\title{
Mutation spectrum leading to an attenuated phenotype in dystrophinopathies
}

\author{
Sylvie Tuffery-Giraud ${ }^{*}{ }^{1}$, Céline Saquet ${ }^{1}$, Delphine Thorel $^{1}$, Antoine Disset ${ }^{1}$, \\ François Rivier ${ }^{2}$, Sue Malcolm ${ }^{3}$ and Mireille Claustres ${ }^{1}$ \\ ${ }^{1}$ Laboratoire de Génétique Moleculaire et Chromosomique, CHU de Montpellier, Montpellier, France; ${ }^{2}$ Service de \\ Neuropédiatrie, Hôpital Gui de Chauliac, CHU de Montpellier, Montpellier, France; ${ }^{3}$ London IDEAS Genetics \\ Knowledge Park, Institute of Child Health, London, UK
}

Although Becker muscular dystrophy (BMD; MIM 300376) is mainly caused by gross deletions of the dystrophin gene, the nature of the mutations involved in the remaining cases is of importance because of the milder clinical course of Becker. We have extensively characterized the mRNA changes associated with five novel point mutations giving rise to a Becker phenotype, which confirm that Becker arises largely due to alterations in splicing. In two cases the milder phenotype arises because of exon skipping, leading to an in-frame deletion (c.1603-2A $>C$ and c.4250T $>A$ ). In further two cases intronic mutations (c.4519-5C $>$ G and c.961-5925A $>C$ ) result in complex splicing changes, but with some residual normal transcripts. The last case, c. 10412T > A (p.Leu3471X), results in a truncated transcript missing only part of the $\mathrm{COOH}$ terminal of the protein, suggesting that this region is not crucial for dystrophin function. The detection of a low amount of dystrophin in this patient could be attributable to a reduced efficiency of nonsensemediated decay. The results emphasize that mRNA analysis is important in defining Becker mutations and will be of value in assessing various gene therapy strategies.

European Journal of Human Genetics (2005) 13, 1254-1260. doi:10.1038/sj.ejhg.5201478;

published online 3 August 2005

Keywords: Becker muscular dystrophy; dystrophin gene; in-frame mutations; transcript analysis; splicing

\section{Introduction}

Duchenne (DMD; MIM 310200) and Becker muscular dystrophy (BMD; MIM 300376) are caused by allelic mutations in the dystrophin gene. In contrast to the more severe Duchenne phenotype, BMD has a more varied and milder clinical course. There is an age of onset from around 12 years, sometimes much later. Patients remain ambulatory beyond the age of 16 years, and a few may even lead near-normal lives. ${ }^{1}$ Death is usually in the fourth or fifth decade. Despite their milder skeletal muscle involvement,

*Correspondence: Dr S Tuffery-Giraud, Laboratoire de Génétique Moléculaire et Chromosomique, Institut Universitaire de Recherche Clinique (IURC), 641 Avenue du Doyen G. Giraud, 34093 Montpellier cedex 5, France. Tel: + 33467415360 ; Fax: + 334674153 65;

E-mail: tuffery@igh.cnrs.fr

Received 23 March 2005; revised 15 June 2005; accepted 30 June 2005; published online 3 August 2005 patients with BMD are at high risk of developing cardiomyopathy, and their most common cause of death is heart failure. ${ }^{2}$ A milder clinical phenotype is correlated with positive staining of muscle fibres, indicating the presence of either a reduced amount of normal dystrophin or the presence of some dystrophin, albeit of altered sequence. ${ }^{3}$ Any molecular genetic mechanism leading to a milder phenotype, or correlation of specific mutations with additional clinical features, is of interest as it may help in the design of strategies for gene therapy.

Deletions of exons of the dystrophin gene are the principle cause of both DMD and BMD. The difference between phenotypes is explained in most cases by the 'frame-shift hypothesis'. ${ }^{4}$ The milder clinical form, BMD, results when the exons deleted correspond to an exact multiple of codons so that some dystrophin, although of altered sequence, is produced. In all, $80 \%$ of Becker cases, 
whose diagnosis was confirmed by dystrophin testing, result from dystrophin deletions ${ }^{1}$ (and our unpublished observations). This is noticeably higher than the $65 \%$ found in DMD, and, together with the fact that BMD occurs less frequently than DMD (1 in 18500 live male births compared to 1 in 4000 in DMD), implies that only a limited range of point mutations are able to act to produce residual dystrophin which can alleviate the severe Duchenne phenotype. Although the Leiden database (www.dmd.nl) contains numerous reports of mutations based on genomic DNA sequencing found in Becker patients, only a few of them have been characterized at the mRNA level and published. ${ }^{5-14}$ Most BMD point mutations disrupt splicing, resulting in an intact but interstitially deleted open reading frame and a partially functional protein. ${ }^{15}$ Without reverse transcription PCR (RT-PCR) analysis, it is not possible to predict the outcome of DNA changes on protein structure $\mathrm{e}^{16,17}$ or to quantitate the various species of mRNA produced by the mutation, including any remaining normal mRNA.

Over the last 10 years, we have provided a comprehensive mutation screening service for DMD and BMD cases referred from various regions of France and Europe. We use a method which analyses for truncated protein products from the entire coding region reverse-transcribed from dystrophin transcripts from muscle biopsy (RT-PCR-protein truncation test (PTT)). ${ }^{18,19}$ Although this strategy is not intended to detect missense mutations, it has the ability to detect deletions or small duplications of exons as well as nonsense mutations, small frame-shift deletions or insertions, and splice mutations. By using this strategy, we previously reported the identification of the diseasecausing mutation in three BMD patients, all of them affecting splicing. ${ }^{12,20}$ Through the extensive analysis of five novel mutations in the dystrophin gene, we demonstrate why, in each case, they led to an attenuated phenotype.

\section{Materials and methods \\ Patients}

Case D306 The patient (born in 1961) was first examined at the age of 40 years. He complained of muscle weakness in the lower limbs and had difficulties in running and climbing stairs. His serum CK was found to be $1414 \mathrm{IU} / \mathrm{l}$ (normal<150 IU/l). Dystrophin Western blot analysis revealed the presence of a reduced amount (about 60\% of normal level) of a normal-sized protein with the antibody against the C-terminal domain (Dys2). He is currently aged 43 years, and the disease is progressing. It is a familial case of dystrophinopathy. Two uncles now deceased became wheelchair bound at age 55 years, and one maternal aunt is symptomatic. He has one brother (aged 29 years), and three cousins (aged 41 and 33 years) also affected. Although they complain of tiredness, pain in the lower legs and have a waddling gait, all of them are still ambulatory and are in employment.

Case D197 The proband (born in 1968) was a 36-year-old man who has suffered from cramps since the age of 7 years. The disease was first diagnosed when blood tests were carried out on entering the army, which revealed elevated CK levels (1607 IU/1; control < $140 \mathrm{IU} / \mathrm{l})$. Calf pseudohypertrophy was also present. He takes part in a lot of sport and, in particular, ran half marathons in 1995 and 1996. To clarify the cause of the elevation in serum CK, a quadriceps muscle biopsy was carried out, which disclosed dystrophic changes on microscopic examination. Immunofluorescence staining for dystrophin revealed a weak signal with all antibodies used, and some fibres were totally negative. These findings clearly indicated dystrophinopathy. He has had clinical signs of dilated myocardiopathy since the age 30 .

Case D282 The clinical course of this patient aged 43 years (born in 1962) clearly shows a Becker phenotype, and he is still ambulatory. The dystrophin analysis by immunofluorescence revealed some normally labelled fibres, whereas some others showed a complete absence of C-terminal domain labelling.

Case D182 The patient (born in 1991) was first examined at 7 years of age because of frequent falling and running difficulties. There was no family history of muscle disease. He started to walk independently at 11 months of age, and his motor development was apparently normal in the first years of life. At the time of first examination (age 7 years and 4 months), he showed a waddling gait, enlarged calves, and was clinically diagnosed with dystrophinopathy based on a highly elevated CK level. Western blot analysis of dystrophin showed the presence of a low amount of a truncated dystrophin with the Dys1 and Dys2 antibodies. His muscle weakness progresses and he has difficulty walking, but he remains ambulant, aged 13 years and 4 months. Patients who lose the ability to walk between age 12 and 16 years are classified as having an intermediate form of dystrophinopathy (IMD), but it is not yet possible to say whether this patient represents a severe Becker phenotype or an intermediate phenotype.

Case D193 Clinical symptoms in this patient (born in 1979) were first observed at 9 years, but the age at which he first walked had been delayed and he has never been able to run fast. The clinical diagnosis was made at 14 years. He has enlarged calves and, now aged 25 years, suffers from hyperlordosis, has some difficulties in walking and has been diagnosed with a dilated cardiomyopathy, whose progression is controlled by medication. Dystrophin Western blot analysis detected the presence of a residual amount of apparently normal size protein (about 5\%). 
Despite the finding of a point mutation in the terminal portion of the gene (see Results), which in other cases has been implicated in mental retardation, his mental development was normal. This patient has obtained the French baccalaureate and has started university.

\section{Mutation analysis}

All tests have been approved by the local ethical committee and informed consent obtained. RT-PCR and PTT were used to analyse the skeletal muscle dystrophin mRNA as described before. ${ }^{19}$ Nucleotide numbers refer to the published cDNA sequence (NM_004006.1), and the mutations have been submitted to the Leiden Muscular Dystrophy mutation database (http://www.dmd.nl/ dmd_all.html). In patient D306, a genomic region that encompasses the inserted intronic sequence detected in the transcripts was amplified using forward $\left(5^{\prime}\right.$-TGCTGTT GCTTAGACTCTTAA-3') and reverse (5'-ACAGATCAGGGT GATAGAAAT-3') primers located in intron 9. The subsequent PCR product was sequenced to identify the nucleotide change, which has led to the inclusion of the cryptic exon in the mature dystrophin transcripts.

\section{Results}

We report five novel point mutations which we have identified by mRNA analysis of non-deletion Becker cases referred through our national referral centre (Table 1). In four cases altered transcripts were observed, but in the fifth case only full-length transcripts were present and the truncated protein was detected by PTT.

Altered mRNA transcripts resulting from changes in splicing were observed in four cases (D306, D197, D282, D182; Figure 1a-d, respectively), with some residual normal full-length transcript in D306 and in D282 (Figure $1 \mathrm{a}$ and $\mathrm{c})$.

\section{Deep intronic mutation}

The mutation in D306 results in activation of a 90-bp pseudoexon coded entirely within intron 9 from positions -5922 to -5832 (GenBank clone AC004468). Sequencing analysis of the genomic region revealed that the patient carries an A-to-C change three bases upstream of the 90-bp insertion, at position -3 of a putative acceptor splice site. This point mutation at position -5925 of intron 9 (c.941$5925 \mathrm{~A}>\mathrm{C}$ ) resulted in the occurrence of a quasi-perfect acceptor splice site, increasing the score $(\mathrm{CV})$ calculated according to Shapiro and Senapathy from 88.3 to $99.7 \% .^{21}$ Splicing between this novel acceptor splice site and one pre-existing, but normally silent, weak donor splice site within intron 9 (CV 73\%) leads to the inclusion of 90 nucleotides in part of the dystrophin transcripts in this patient. A stop codon occurs at the seventh amino-acid residue within the insertion, resulting in a truncated transcript. In accordance with the dystrophin results, RT-PCR shows that a large amount of the normal transcript is still detectable (Figure 1a).

\section{Mutations at consensus splice sites}

Case D197 has a mutation $(A>C)$ at the invariant AG within the splice acceptor site of intron 14 . This results in two mRNA species, an in-frame deletion of exon 14 and a deletion of exon 14 plus exon 15 in a minor population of transcripts (Figure 1b), which is also in-frame. Thus, this mutation, although it is a splicing mutation, fulfils the predictions of the reading frame rule.

The mutation in case D282 involves a $C$ to $G$ change at position -5 of the acceptor splice site of intron 32 . This falls within the pyrimidine-rich region from -14 to -5 . Position -5 is by no means invariant, but G occurs in only $8 \%$ of primate splice sites compared to C in $40 \%$. The weak wild-type CV score (69.40\%) is further reduced to $64.40 \%$. It has several effects (Figure 1c): in part it causes a deletion of exon 33, which produces an in-frame product in a minor

Table 1 Point mutations identified in Becker patients by transcript analysis

\begin{tabular}{|c|c|c|c|c|}
\hline Patient & Age & Mutation & Effect on transcripts & Dystrophin analysis \\
\hline D306 & 43 years & c. $961-5925 A>C$ & $\begin{array}{l}\text { ins } 90 \text { bp from intron } 9 \text { full- } \\
\text { length transcripts }\end{array}$ & $\begin{array}{l}\text { WB: } 60 \% \text { of normal level, } \\
\text { normal-size protein }\end{array}$ \\
\hline D197 & 36 years & c. $1603-2 A>C$ & $\begin{array}{l}\text { Skipping of exon } 14(\mathrm{InF}) \\
\text { skipping of exon } 14+15(\mathrm{InF})\end{array}$ & $\begin{array}{l}\text { IF: weak dystrophin staining } \\
\text { with Dys1, Dys2, Dys } 3\end{array}$ \\
\hline D282 & 42 years & c. $4519-5 C>G$ & $\begin{array}{l}\text { Skipping of exon } 33 \text { (InF) ins } \\
4 \text { bp from intron } 32 \text { full- } \\
\text { length transcripts }\end{array}$ & $\begin{array}{l}\text { IF: mosaic pattern of dystrophin } \\
\text { staining with Dys } 2\end{array}$ \\
\hline D182 & $\begin{array}{l}13 \text { years and } \\
4 \text { months }\end{array}$ & $\begin{array}{c}\text { c.4250T >A } \\
(\text { p.Leu1417X) }\end{array}$ & $\begin{array}{l}\text { Full-length transcripts } \\
\text { skipping of exon } 31 \text { (InF) }\end{array}$ & $\begin{array}{l}\text { WB: truncated dystrophin of } \\
\text { reduced quantity (faint band } \\
\text { detected with Dys } 2 \text { ) }\end{array}$ \\
\hline D193 & 25 years & $\begin{array}{l}\text { c. } 10412 T>A \\
(p . L e u 3471 X)\end{array}$ & Full-length transcripts & $\begin{array}{l}\text { WB: } 5 \% \text { of normal level, } \\
\text { apparently normal size }\end{array}$ \\
\hline
\end{tabular}

InF: in-frame; IF: immunofluorescence analysis; WB: Western blot analysis. 


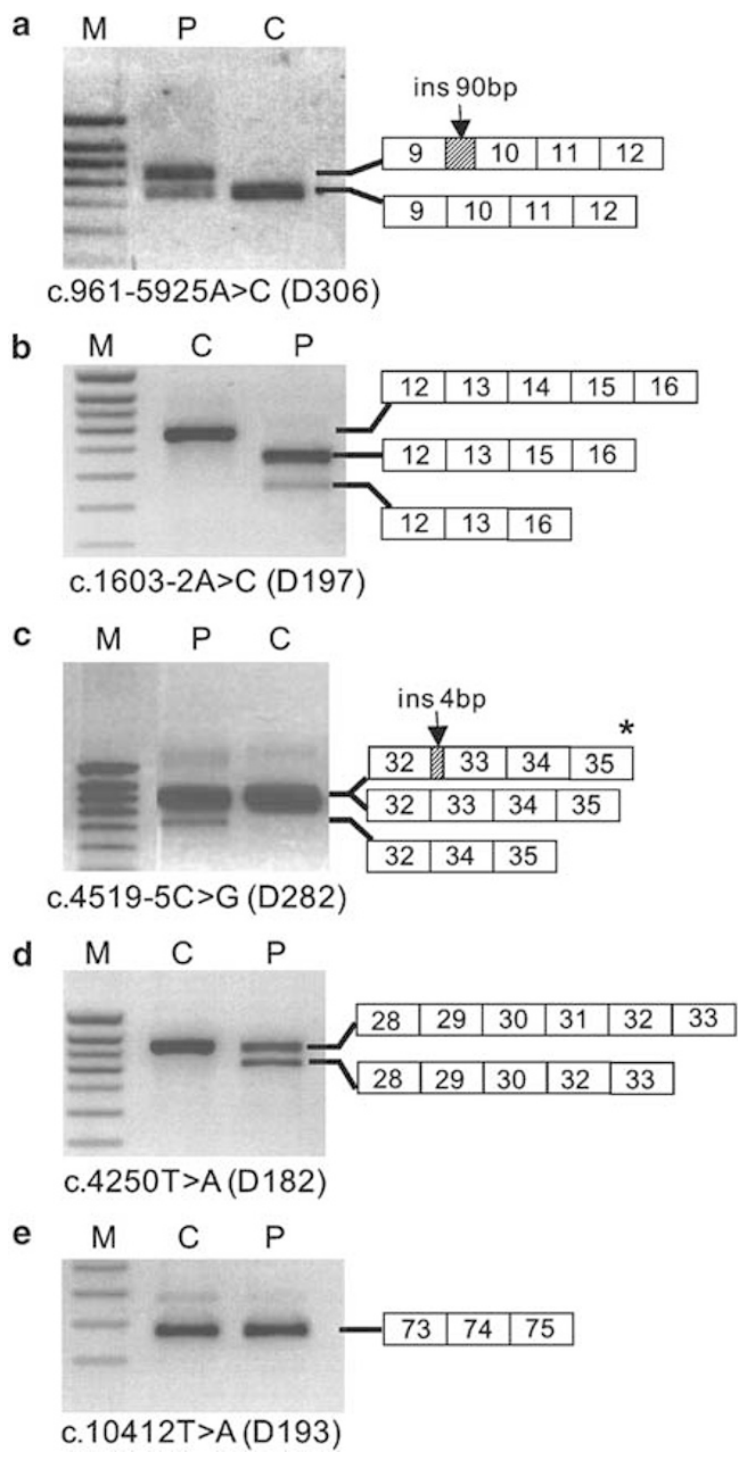

Figure 1 Dystrophin transcripts analysis. Amplified fragments were separated on a $2 \%$ agarose gel. M: molecular weight marker, C: control, P: patient. The schematic representation of the resulting transcripts is reported. E: $\left(^{*}\right)$ the asterisk indicates that the difference in size between the normal full-length fragment and the transcript with the four intronic base pairs insertion could not be resolved in the patient $(P)$.

population of transcripts and, also, by creating a new ag dinucleotide at position -6 to -5 , activates a nearby cryptic splice site four bases upstream with a high Shapiro and Senapathy score of $81.7 \%$ in the vast majority of transcripts (which leads to an out of frame product). Some very low level of normal transcript could also be detected by sequence analysis of the transcript products. The residual dystrophin from the normal transcripts and the product with exon 33 deleted are both predicted to lead to a Becker phenotype.

\section{Mutations in the coding sequence}

In patient D182, in addition to the full-length transcript which harbours the c.4250T $>$ A (p. Leu1417X) nonsense mutation in exon 31, a shorter product is found, sequencing of which showed that it resulted from skipping of exon 31 (Figure 1d). This results in an in-frame deletion consistent with the outcome of a milder phenotype, and presumably arises from disruption of an exonic splice enhancer (ESE), a motif involved in exon definition, which when disrupted affects the splicing profile of transcripts. The exon skipping may also result from the creation of an exonic splicing silencer (ESS), which would inhibit in that case the recognition of the exon.

Case D193 carries a nonsense mutation c.10412T $>$ A (p.Leu3471X) in exon 74. The truncated protein was detected by PTT. Several assays of muscle dystrophin transcripts amplification using different combinations of primers could not detect any skipping event of exon 74 (Figure 1e) or exons 71-74, which would have eliminated the mutation and generated in-frame transcripts. Only skipping of exon 71 was detected at a low level in the muscle transcripts (data not shown). The possibility of either exon skipping or aberrant splicing thus was ruled out. Therefore, the discrepancy between genotype and phenotype could not be explained at the mRNA level. Although Western blotting detected reduced amounts of apparently normal protein, a small difference in size could not have been not detected by the standard techniques used. Also, a somatic mosaicism could be excluded in this patient as his mother was found to be a carrier of the mutation.

\section{Discussion}

The mutations described in this study lead to several molecular mechanisms, which attenuate the severe phenotype of DMD. Most mutations giving rise to BMD are the result of deletions of an exon or exons which result in no frame shift. ${ }^{4}$ Two of the mutations presented here also result in mRNA with internal in-frame deletions, but via a splicing mechanism.

In one case, D182, a nonsense mutation at amino acid 1417, results in exon skipping. The same mutation resulting in a BMD phenotype is described in the Leiden database (www.dmd.nl), but without any RNA studies. Examples of exon skipping have been reported in nonsense mutations identified in exons 25, 27, 29 and 72 of the dystrophin gene. ${ }^{9,10,22,23}$ Mutation-associated exon skipping is recognized in an increasing number of genes as a novel form of splicing mutations. ${ }^{24}$ These mutations most likely induce exon skipping by affecting cis-acting elements which interact directly or indirectly with trans-acting activators or repressors of splicing. Whereas exons are activated by $\operatorname{ESEs}^{25}$ which are recognized by 


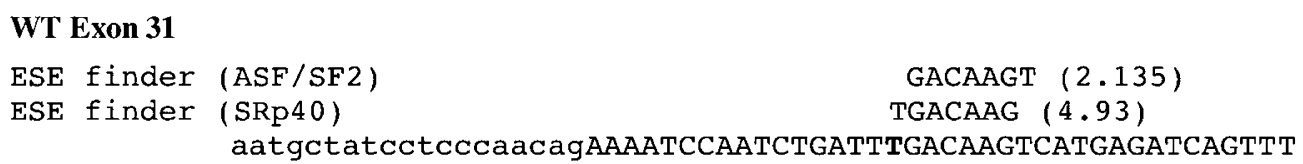

aatgctatcctcccaacagAAAATCCAATCTGATTTGACAAGTCATGAGATCAGTTT

\begin{tabular}{|c|c|}
\hline Mutant Exon 31 & \\
\hline ESE finder (ASF/SF 2) & GACAAGT $(2.135)$ \\
\hline ESE finder (SRp40) & AGACAAG $(3.38)$ \\
\hline RESCUE-ESE & AGACAA \\
\hline aatgct & $\begin{array}{l}\text { TAGACAAGTCATGAGATCAGTTT } \\
\text { * }\end{array}$ \\
\hline FAS-ESS (hex2) & TAG \\
\hline
\end{tabular}

Figure 2 Effect of the C.4250T > A mutation on putative ESE and ESS elements. The intron sequence is in lowercase, and the exon sequence is in uppercase (only the first nucleotides of exon 31 are shown). The position of the C.4250T >A mutation is indicated by a star. The presence of hexamers predicted by RESCUE-ESE (http://genes.mit.edu/burgelab/rescue-ese/) to function as ESE elements and the scores for individual candidate SR proteinbinding sites calculated by ESE finder (http://exon.cshl.org/ESE) are indicated above the sequences. The prediction for the identification of candidate ESS hexameric sequences (http://genes.mit.edu/fas-ess/) is reported below the sequences. Only predicted motifs at the immediate vicinity of the mutation are represented.

serine/arginine-rich (SR) proteins, a family of essential splicing factors, they are repressed by ESSs. ${ }^{26}$ To test whether the c.4250T $>$ A exon-skipping mutation in exon 31 either abrogates an ESE or creates an ESS, we used available prediction programs: ESEfinder ${ }^{25}$ and RESCUE-ESE ${ }^{27}$ for enhancers and the FAS-ESS for silencers, to analyse the wildtype and mutant sequences of exon 31 (Figure 2). The nonsense mutation was found to decrease an SRp40 motif (4.93 to 3.38) according to ESEfinder, but to create a novel ESE hexamer according to RESCUE-ESE. The mutation does not affect a partially overlapping ASF/SF2 motif. Moreover, the FAS-ESS predicted the creation of a silencer motif by the mutation. Hence, the in silico approach indicates the presence of several potentially splicing regulating sequences in exon 31. However, the reliability of this prediction and the full characterization of the mechanism underlying the partial exon skipping observed in patient D182 would require further functional studies.

The second splice mutation (c.1603-2A >C) leading to exon skipping and an in-frame deletion resulted from a straightforward mutation in an invariant ' $a$ ' at the -2 position of the intronic acceptor site. However, RNA analysis showed two altered species resulting from skipping of exon 14 and exon 14 plus 15 in a subpopulation of transcripts. Both of these deletions lead to an in-frame product which can contribute to the Becker phenotype, and it again shows the importance of direct RNA measurements to detect minor species. In accordance with the detection of exclusively in-frame transcripts in this patient, the immunocytochemical analysis of dystrophin revealed a weak staining of all the fibres with the antibodies used. Several studies have indicated that patients with BMD also have a high incidence of clinical cardiac involvement despite their milder skeletal muscle disease. It was demonstrated that, in a series of 68 patients whose diagnosis was confirmed by dystrophin testing, all had preclinical or clinical cardiac involvement by the age of $30 .^{2,28}$ This is also the case in patient D197, who complains of clinical signs of cardiomyopathy since the age of 30 years.

D282 and D306 provide examples of a further class of mutation: those within introns whose effect on transcript structure may be difficult to predict. In D282 the mutation is at position -5 of intron 32 and could have been picked up by analysis of genomic DNA with the right choice of PCR primers, although the significance would have been unclear. The wild-type acceptor site of intron 32 is already a weak site, with several deviations from the consensus sequence (A's at positions $-6,7$ and 8 ). The mutation $C>G$ at position -5 not only weakens it further so that exon 33 is skipped, but also results in creation of a cryptic splice site 4 nucleotides upstream of the IVS 32 splice junction with a higher consensus value (CV 81.7\%). This new acceptor site is widely used in the transcripts of the patient. Some fulllength normal RNA is also produced. The cryptic site results in a frame-shifted product, but both the exon 33 skipped product and the reduced amount of normal product will contribute to the milder phenotype. The identification of both out-of-frame and in-frame dystrophin mRNA was consistent with the immunofluorescence staining for dystrophin in this patient, which disclosed a mosaic pattern of dystrophin expression with some fibres normally labelled and others completely negative.

D306 has a mutation $\mathrm{A}>\mathrm{C}$ at position -5925 of intron 9. This creates a novel acceptor site, leading to the insertion of a 90-bp cryptic exon. The $\mathrm{C}$ occurs at position -3 of this pseudoexon, which is closer to the consensus sequence (74\%) than A (3\%). However, the donor splice site would normally be expected to be weak as it breaks the ' $\mathrm{gt}^{\prime}$ ' splice donor rule with a ' $\mathrm{gc}^{\prime}$. This is the same pseudoexon as described in case \#3 in Beroud et al, ${ }^{14}$ in which that ' $\mathrm{gc}^{\prime}$ ' was 
mutated to ' $\mathrm{gt}$ '. Interestingly, in that case, the pseudoexon uses the same acceptor site as described here, without mutation. This implies that the acceptor site is fairly strong in any case, but is improved with the $\mathrm{A}>\mathrm{C}$ change. It would be extremely difficult to predict in advance the consequence of these changes, and, coupled with the fact that in these and other reported cases mutations were found deep into introns, ${ }^{12,14}$ it would be very difficult to detect them and assess their significance without RNA studies. Although the cases result from the activation of pseudoexons, the Becker phenotype arises because the mutations do not affect the coding sequence and there is residual dystrophin produced from the remaining normal transcript.

The final mutation (p.Leu3471X) is the result of a truncating mutation which occurred close to the $\mathrm{COOH}$ terminus of the protein. The patient, at the time of writing, was 25 years old, with a phenotype clearly milder than DMD. He has above-average intelligence, but has been diagnosed with dilated cardiomyopathy. Nonsense mutations almost always result in DMD and are very uncommon in BMD. However, the Leiden database shows a cluster of truncating mutations in exon 74 extending from Leu3471 (this paper) to Glu3515, and including amino-acid positions Leu3485, Gln3493, Leu3485X3495 (due to the mutation c.10454delT), Gln3493fsX3495 (due to the mutation c.10477delC) and Ser3500. Only in the case reported here and a few of the previously described mutations (c.10454del, ${ }^{29}$ and c.10477delC ${ }^{30}$ ) have RNA studies been carried out and the absence of normal RNA or alternative splicing of the in-frame exon 74 been demonstrated. Although there is one exception with the recent description of an asymptomatic boy carrying the p.Glu3625X nonsense mutation in exon $76,{ }^{31}$ nonsense mutations in the last two exons, 75 and 76, again give rise to a DMD phenotype. Variability in the efficacy of NMD has been proposed as a possible explanation for the great phenotypic variability observed between patients harbouring truncating mutations $3^{\prime}$ of exon 70 in the dystrophin gene. ${ }^{32}$ Nonsense-mediated mRNA decay in mammalian cells generally occurs when a nonsense codon resides >50-55 nucleotides upstream of a splicing-generated exon-exon junction. On the basis of the current knowledge, the efficiency of NMD is generally not influenced by nonsense codon position, indicating that a higher number of downstream exon junction complex (EJC) of proteins does not lead to more efficient NMD. ${ }^{33}$ However, might a low number of EJCs induce a lower efficiency of NMD? Data of transcript and dystrophin analysis in a series of BMD patients carrying C-terminally truncating mutations would argue for this hypothesis, ${ }^{32}$ and the low amount of dystrophin detected in patient D193 could be attributable to the reduced efficacy of NMD. In contrast with the BMD patient reported by Kerr et $a^{32}$ who carries the c. 10454delT mutation in exon 74 and presented with mental retarda- tion, the truncated dystrophin in patient D193 allows rescue of both his muscle and cognitive phenotype. Hence, the variability of NMD efficiency would not affect similarly the tissue-specific dystrophin isoforms, and this would differ between individuals.

In accordance with previous studies, the partial rescue of the phenotype in patient D193 sustains the hypothesis that the p.Leu3471X mutation does not lie within a protein domain crucial for the normal function of the full-length and shorter isoforms of dystrophin. ${ }^{32,34}$ The dystrophin protein carries out its role in maintaining muscle fibres intact by binding via the $\mathrm{N}$-terminal end to the subsarcolemmal cytoskeletal actin network, and via its cysteine-rich domain to $\beta$-dystroglycan. The above results suggest that the amino-acid residues 3471-3500 are not essential for this role. This is consistent with binding studies which showed that residues 3026-3345 were essential for binding. ${ }^{35}$ In addition to a structural role, the dystrophin complex acts as a scaffold that concentrates and stabilizes functionally inter-related signalling proteins at the muscle cell membrane. Signalling function of the dystrophin complex is mediated by the binding of dystrobrevin and syntrophin in the COOH-terminal region of dystrophin. ${ }^{36}$ Nevertheless, the absence of this region is compatible with a moderate phenotype in our patient as previously reported. ${ }^{32,34}$

Many different strategies for DMD gene therapy have been studied and improved over the years. One promising development concerns the induction of exon-skipping events with small molecules that affect dystrophin gene splicing in order to restore the reading frame. ${ }^{37}$ Such genetic approaches are expected to produce an attenuated Becker-like phenotype as observed in the reported patients of our study.

\section{Acknowledgements}

We are grateful to the physicians (Drs V HumbertClaude, $C$ Coubes, $M$ Cossée, M Koenig, C Philippe, $K N^{\prime}$ Guyen, F Leturcq) for referral of subjects and clinical data or dystrophin analysis. AD is supported by a grant of Association Française contre les Myopathies (AFM).

\section{References}

1 Bushby KM, Gardner-Medwin D, Nicholson LV et al: The clinical, genetic and dystrophin characteristics of Becker muscular dystrophy. II. Correlation of phenotype with genetic and protein abnormalities. J Neurol 1993; 240: 105-112.

2 Nigro G, Comi LI, Politano L et al: Evaluation of the cardiomyopathy in Becker muscular dystrophy. Muscle Nerve 1995; 18: 283-291.

3 Nicholson LV, Johnson MA, Bushby KM et al: Integrated study of 100 patients with Xp21 linked muscular dystrophy using clinical, genetic, immunochemical, and histopathological data. Part 3. Differential diagnosis and prognosis. J Med Genet 1993; 30: $745-751$.

4 Monaco AP, Bertelson CJ, Liechti-Gallati S, Moser H, Kunkel LM: An explanation for the phenotypic differences between patients 
bearing partial deletions of the DMD locus. Genomics 1988; 2: 90-95.

5 Roberts RG, Passos-Bueno MR, Bobrow M, Vainzof M, Zatz M: Point mutation in a Becker muscular dystrophy patient. Hum Mol Genet 1993; 2: 75-77.

6 Hagiwara Y, Nishio H, Kitoh Y et al: A novel point mutation (G-1 to T) in a $5^{\prime}$ splice donor site of intron 13 of the dystrophin gene results in exon skipping and is responsible for Becker muscular dystrophy. Am J Hum Genet 1994; 54: 53-61.

7 Bartolo C, Papp AC, Snyder PJ et al: A novel splice site mutation in a Becker muscular dystrophy patient. J Med Genet 1996; 33: 324-327.

8 Patria SY, Alimsardjono H, Nishio H, Takeshima Y, Nakamura H, Matsuo M: A case of Becker muscualr dystrophy resulting from the skipping of four contiguous exons (71-74) of the dystrophin gene during mRNA maturation. Proc Assoc Am Physicians 1996; 108: $308-314$

9 Shiga N, Takeshima Y, Sakamoto H et al: Disruption of the splicing enhancer sequence within exon 27 of the dystrophin gene by a nonsense mutation induces partial skipping of the exon and is responsible for Becker muscular dystrophy. J Clin Invest 1997; 100: 2204-2210.

10 Fajkusova L, Lukas Z, Tvrdikova M, Kuhrova V, Hajek J, Fajkus J: Novel dystrophin mutations revealed by analysis of dystrophin mRNA: alternative splicing suppresses the phenotypic effect of a nonsense mutation. Neuromuscul Disord 2001; 11: 133-138.

11 Cagliani R, Bardoni A, Sironi M et al: Two dystrophin proteins and transcripts in a mild dystrophinopathic patient. Neuromusc Disord 2003; 13: 13-16.

12 Tuffery-Giraud S, Saquet S, Chambert S, Claustres M: Pseudoexon activation in the DMD gene as a novel mechanism for Becker muscular dystrophy. Hum Mut 2003; 21: 608-614.

13 Adachi K, Takeshima Y, Wada H, Yagi M, Nakamura H, Matsuo M: Heterogous dystrophin mRNA produced by a novel splice acceptor site mutation in intermediate dystrophinopathy. Pediatr Res 2003; 53: 125-131.

14 Bëroud C, Carrië A, Beldjord C et al: Dystrophinopathy caused by mid-intronic substitutions activating cryptic exons in the DMD gene. Neuromusc Disord 2004; 14: 10-18.

15 Robert RG, Gardner RJ, Bobrow M: Searching for the 1 in 2400 000: a review of dystrophin gene point mutations. Hum Mutat 1994; 4: 1-11.

16 Flanigan KM, von Niederhausern A, Dunn DM, Alder J, Mendell JR, Weiss RB: Rapid direct analysis of the dystrophin gene. Am J Hum Genet 2003; 72: 931-939.

17 Hofstra RMW, Mulder IM, Vossen R et al: DGGE based whole gene mutation scanning of the dystrophin gene in Duchenne and Becker muscular dystrophy patients. Hum Mutat 2004; 23: 57-66.

18 Roest PAM, Roberts RG, Sugino S, van Ommen G-JB, den Dunnen JT: Protein truncation test (PTT) for rapid detection of translation - terminating mutations. Hum Mol Genet 1993; 2: 1719-1721.

19 Tuffery-Giraud S, Saquet C, Chambert S et al: The role of muscle biopsy in analysis of the dystrophin gene in Duchenne muscular dystrophy: experience of a national referral centre. Neuromusc Disord 2004; 14: 650-658.

20 Tuffery-Giraud S, Chambert S, Demaille J, Claustres M: Point mutations in the dystrophin gene: evidence for frequent use of cryptic splice sites as a result of splicing defects. Hum Mutat 1999; 14: $359-368$.

21 Shapiro MB, Senepathy P: RNA splice junctions of the different classes of eukaryotes: sequence statistics and functional implications in gene expression. Nucleic Acids Res 1987; 15: 7155-7174.

22 Melis MA, Muntoni F, Cau $\mathrm{M}$ et al: Novel nonsense mutation (C->A nt 10512) in exon 72 of dystrophin gene leading to exon skipping in a patient with a mild dystrophinopathy. Hum Mutat 1998; (Suppl 1): S137-S138.

23 Ginjaar IB, Kneppers AL, v d Meulen JD et al: Dystrophin nonsense mutation induces different levels of exon 29 skipping and leads to variable phenotypes within one BMD family. Eur $J$ Hum Genet 2000; 8: 793-796.

24 Faustino NA, Cooper TA: Pre-mRNA splicing and human disease. Genes Dev 2003; 17: 419-437.

25 Cartegni L, Wang J, Zhu Z, Zhang MQ, Krainer AR: ESEfinder: a web resource to identify exonic splicing enhancers. Nucleic Acid Res 2003; 31: 3568-3571.

26 Wang Z, Rolish ME, Yeo G, Tung V, Mawson M, Burge CB: Systematic identification and analysis of exonic splicing silencers. Cell 2004; 119: 831-845.

27 Fairbrother WG, Yeh RF, Sharp PA, Burge CB: Predictive identification of exonic splicing enhancers in human genes. Science 2002; 297: 1007-1013.

28 Melacini P, Fanin M, Danieli GA et al: Myocardial involvement is very frequent among patients affected with subclinical Becker's muscular dystrophy. Circulation 1996; 94: 3168-3175.

29 Roberts RG, Bobrow M, Bentley DR: Point mutations in the dystrophin gene. Proc Natl Acad Sci USA 1992; 89: 2331-2335.

30 Gardner RJ, Bobrow M, Roberts RG: The identification of point mutations in Duchenne muscular dystrophy patients by using reverse transcription PCR and the protein truncation test. Am J Hum Genet 1995; 57: 311-320.

31 Suminaga R, Takeshima Y, Wada H, Yagi M, Matsuo M: C-terminal truncated dystrophin identified in skeletal muscle of an asymptomatic boy with a novel nonsense mutation of the dystrophin gene. Pediatr Res 2004; 56: 739-743.

32 Kerr TP, Sewry CA, Robb SA, Roberts RG: Long mutant dystrophins and variable phenotypes: evasion of nonsensemediated decay. Hum Genet 2001; 109: 402-407.

33 Maquat LE: Nonsense-mediated mRNA decay in mammals. J Cell Sci 2005; 118: $1773-1776$.

34 Crawford GE, Faulkner JA, Crosbie RH et al: Assembly of the dystrophin-associated protein complex does not require the dystrophin COOH-terminal domain. I Cell Biol 2000; 150: 1399-1410.

35 Ishikawa-Sakurai M, Yoshida M, Imamura M, Davies KE, Ozawa E: $\mathrm{ZZ}$ domain is essentially required for the physiological binding of dystrophin and utrophin to beta-dystroglycan. Hum Mol Genet 2004; 13: 693-702.

36 Newey SE, Benson MA, Ponting CP, Davies KE, Blake DJ: Alternative splicing of dystrobrevin regulates the stoichiometry of syntrophin binding to the dystrophin protein complex. Curr Biol 2000; 10: 1295-1298.

37 Goyenvalle A, Vulin A, Fougerousse F et al: Rescue of dystrophic muscle through U7 snRNA-mediated exon skipping. Science 2004; 306: 1796-1799. 